\title{
A multi-objective Pareto ant colony algorithm for the Multi-Depot Vehicle Routing problem with Backhauls
}

\author{
Jhon Jairo Santa Chávez ${ }^{\mathrm{a}}$, John Willmer Escobar ${ }^{\mathrm{b} *}$ and Mauricio Granada Echeverri ${ }^{\mathrm{c}}$
}

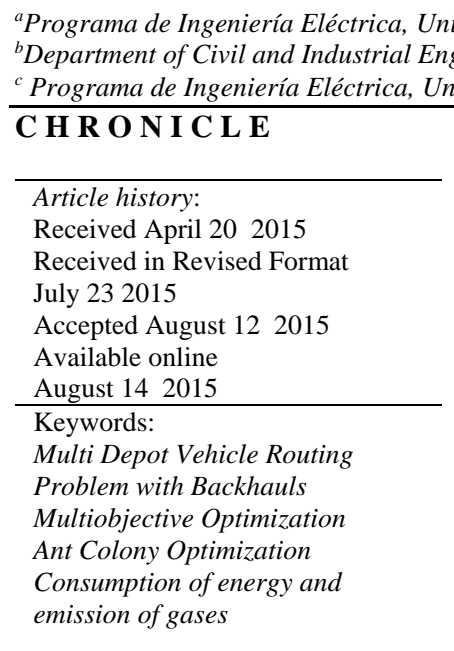

\section{Introduction}

The Multi-Depot Vehicle Routing Problem with Backhauls (MDVRPB) is an operational problem of the supply chain management. The MDVRPB considers a supply chain involving two echelons: depots and customers. The MDVRPB is an NP-hard problem, since it is a generalization of the two well-known NPhard problems: the Multi-Depot Vehicle Routing Problem (MDVRP) (for further details see Escobar et al. 2014a) and the Vehicle Routing Problem with Backhauls (VRPB). The MDVRPB has many realistic applications in Transportation and Logistics. The features of the customers, depots and vehicles, as well as different operating constraints on the performed routes, leads to different variants of MDVRPB: (i) simultaneous collecting and dispatching of products; (ii) collecting first, following the delivery of products; and (iii) collecting after of the delivering process. We have considered a specific version for which the collection of products must be performed after the backhaul customers have been served.

* Corresponding author.

E-mail: jwescobar@javerianacali.edu.co (J. W. Escobar)

(c) 2016 Growing Science Ltd. All rights reserved. doi: 10.5267/j.ijiec.2015.8.003 
The MDVRPB can be defined as the following graph theory problem. Let $G=(V, A)$ be a complete undirected graph, where $V=\{1 \ldots \ldots v\}$ is the set of vertices, and $A$ is the set of edges. The set $V$ is partitioned into two subsets: the set of customers $N=\{1 \ldots n\}$ and the set of potential depots $M=$ $\{1 \ldots \ldots m\}$. Additionally, the set $N$ is divided into a subset of Linehaul nodes (Linehaul customers $-L$ ), and the Backhaul nodes (Backhaul customers $-B$ ). Therefore, $N=L \cup B$. The Linehaul customers ask for delivering products while Backhaul customers require the collection of products. Each customer has a nonnegative amount $D_{i}(j \in N)$ of product to be delivered $(i \in L)$ or to be picked up $(i \in B)$. Each depot has a fictitious demand. i.e. $D_{i}=0$, with $i \in M$. A set of $K$ identical vehicles with a given capacity $Q_{k}$ is initially placed at each depot. It must be clarified that all vehicles are not necessarily used. For each edge $(i, j) \in A$, a nonnegative cost $c_{i j}$ is associated, where $c_{i i}=+\infty$ for each edge $(i, i) \in A, i \in M$. As the symmetrical condition is assumed, for each edge $(i, j) \in V$ the cost of $c_{i j}$ is equal to $c_{j i}$, for all $i \neq j$. In the MDVRPB, all deposits are not necessarily used. The main goal of the MDVRPB is to find a set of $r$ performed routes by imposing the following constraints:

- Each vehicle must start from a depot $m$, and return to the same depot;

- Each customer must be visited exactly once.

- The sum of the demands of customers belonging to a determined route must not exceed the vehicle capacity $Q$;

- For each performed route, the Linehaul customers must precede the Backhaul customers for each sequence;

- The flow between depots is not allowed.

The goal of the MDVRPB is to determine the routes to be performed from the selected depots to the customers by a fleet of homogeneous vehicles in order to satisfy the demand of the customers (products to be collected or products to be delivered). The objective functions considered for the multiobjective version of the MDVRPB is to minimize the total traveled distance, the total time and the consumed energy. The first objective is the common function considered in the literature related to the vehicle routing problems. The second objective is obtained by the allowed speed on each edge. In particular, we have considered a random speed between $30 \mathrm{~km} / \mathrm{hr}$ to $90 \mathrm{~km} / \mathrm{hr}$ for the complete graph on the benchmarking set of instances. Finally, the third objective is adopted from the idea of gas emission and consumption of energy introduced by Bektaş and Laporte (2011) and Demir et al. (2014).

In particular, the consumption of energy $\left(P_{i j}\right)$ for each edge $(i, j)$ is calculated following the idea introduced by Bektaş and Laporte (2011):

$$
P_{i j} \approx \propto_{i j}\left(\xi+f_{i j}\right) d_{i j}+\beta v_{i j}^{2} d_{i j}
$$

where $\propto_{i j}$ is a parameter of the edge $(i, j)$, $\xi$ is the mass of the empty vehicle in kg; $f_{i j}$ is the quantity of product carried from $i$ to $j$ in $\mathrm{kg}$, and $d_{i j}$ corresponds to the distance between $i$ and $j$ in $m$. In addition, $\beta$ is a constant and $v_{i j}^{2}$ corresponds to the speed of the vehicle on edge $(i, j) . \propto_{i j}$ and $\beta$ are obtained as follow:

$$
\begin{aligned}
& \propto_{i j}=a+g \sin \theta_{i j}+g C_{r} \cos \theta_{i j}, \\
& \beta=0.5 C_{d} A \rho,
\end{aligned}
$$

where $a$ corresponds to acceleration of the vehicle in $\mathrm{m} / \mathrm{s}^{2}, g$ is the acceleration of gravity in $\mathrm{m} / \mathrm{s}^{2}, \theta_{i j}$ is the angle of the edge $(i, j), C_{r}$ is the friction coefficient between the vehicle and the road, $C_{d}$ is the drag coefficient, $A$ is the frontal area of the vehicle in $\mathrm{m}^{2}$ and $\rho$ is the density of the air in $\mathrm{kg} / \mathrm{m}^{3}$. 
Salhi and Nagy (1999) proposed a heuristic approach to solve the MDVRPB, which is is based on the idea of "Border Customers", defined as the customers geographically located in a middle point between two depots. Min et al. (1992) introduced a version of the MDVRPB by considering collection process after delivery of products. A unified heuristic for different vehicle routing problems with backhaul was presented by Ropke and Pisinger (2006). In this work, a version of mixed pickup and delivery with multiple depots was considered. Two algorithms based on ant colony for the multi depot vehicle routing problem with mixed pickup and delivery were presented by Wade and Salhi (2004) and Wade and Salhi (2001). Finally, genetic algorithms for solving the MDVRPB were proposed by Chunyu and Xiaobo (2009) and Chunyu et al. (2009).

Surveys of existing methods for multi-objective problems were presented in Jozefowiez et al. (2008) and Zhou et al. (2011). In Jozefowiez et al. (2008), the authors examined multiobjective versions of several variants of the Vehicle Routing Problem (VRP) in terms of their objectives, their characteristics and the types of proposed algorithms to solve them. A survey of the state of the art of the multi-objective evolutionary algorithms was proposed by Zhou et al. (2011). This papers covers algorithms frameworks for multiobjective combinatorial problems during the last eight years. However, in the literature reviewed, there are few works considering the multi-objective version of the MDVRPB. Multiobjective metaheuristic approaches for combinatorial problems were presented in Doerner et al. (2004), Liu et al. (2006) and Lau et al. (2009). A multiobjective methodology by Pareto Ant Colony Optimization for solving a portfolio problem was introduced by Doerner et al. (2004). A multi-objective mixed zero-one integer-programming model for the vehicle routing problem with balanced workload and delivery time was introduced by Liu et al. (2006). In this work, a heuristic-based solution method was developed. A fuzzy multi-objective evolutionary algorithm for the problem of optimization of vehicle routing problems with multiple depots, multiple customers, and multiple products was proposed by Lau et al. (2009). In this work, two objectives were considered: minimization of the traveling distance and also the traveling time.

Approaches for multi-objective versions of the VRPB have been proposed by Anbuudayasankar et al. (2012), García-Nájera et al. (2015) and Yalcın \& Erginel (2015). Three heuristics approaches for solving a bi-objective vehicle routing problem with forced backhauls were introduced by Anbuudayasankar et al. (2012). In particular, two heuristics are based on the well-known savings algorithm and the third heuristic is based on a Genetic Algorithm (GA). Finally, an evolutionary approach and a fuzzy programming for the multi-objective vehicle routing problems with backhauls were presented by GarcíaNájera et al. (2015) and Yalcin and Erginel (2015), respectively. Other multiobjective algorithms proposed for solving related logistic combinatorial problems could be consulted in Nezhad et al. (2013), Mortezaei and JabalAmeli (2011), Mohammadi et al. (2011), Rao and Patel (2014), Yazdian and Shahanaghi (2011), Escobar et al. (2013), Escobar et al. (2014b), Escobar et al. (2015) and Bolaños et al. (2015).

This paper proposes a multiobjective algorithm based on an Ant Colony System to solve the MDVRPB with collection of products exclusively after delivery of products. The efficiency of the proposed algorithm has been compared by 33 modified instances taken from the literature. The set of instances originally was proposed by Salhi and Nagy (1999). In particular, information about of mitigation of gas emissions, consumption of energy and traveling times has been added to the set of instances.

The main contribution of the paper is to propose an effective algorithm for the solution of a multiobjective version of the multi-depot vehicle routing problem with backhauls (MDVRPB). The proposed algorithm is a novel metaheuristic approach, which obtains good results within short computing times. This version of the MDVRPB has not been considered in the previous revised literature. Therefore, no attempt has been proposed for solving this variant of the problem. 
This paper is organized as follows: Section 2 provides a description of the general framework of the proposed approach. Section 3 gives a detailed description of the proposed multi-objective metaheuristic approach for the MDVRRPB. Section 4 described the analysis of computational results. Finally, section 5 shows the conclusions and future research.

\section{Metaheuristic algorithm based on ant colony system}

The Ant Colony metaheuristic is based on the natural behavior of ants searching food. The logical tendency of each ant is to reduce the effort and time required to obtain food. This goal is achieved by reducing the distance, time and energy between two specific points for collecting food. The ants are individuals with relatively simple features. However, they perform highly complex work in a simple way. The success lies in the interaction of many individuals with the environment and by indirect communication between them through chemicals substances called pheromones (Gutjahr, 2002). This behavior is emulated in artificial intelligence to find good quality solutions to combinatorial optimization problems characterized by a wide space of solutions.

\subsection{Selecting edges}

The proposed algorithm considers an ant as the emulation of a vehicle in the process of performing a route. An artificial ant begins its travel from a depot selected randomly to a first customer. The decision of the next client to be visited is based on heuristic preferences biased by the distance, time and energy among nodes and the emulation of the natural pheromone. A probabilistic transition rule defines the likelihood that the ant $k$ (one ant for each available vehicle), placed at node $i$, decides to move to the node $j$ (selecting the edge $(i, j) \in A$ ). The rule is defined according to the Eq. (4).

$$
p_{k}(i . j)=\frac{\left[\tau_{i j}\right]^{\alpha} \cdot\left[\eta_{i j}\right]^{\beta}}{\sum_{u \in S}\left[\tau_{i j}\right]^{\alpha} \cdot\left[\eta_{i j}\right]^{\beta}},
$$

where $\tau_{i j}$ is the amount of pheromone on the edge $(i . j) \in A$, and $\eta_{i j}$ is the heuristic information of $(i . j)$, i.e. the distance, time and energy to travel from the node $i$ to the node $j$. The level of importance on the rule decision from both the heuristic information and the amount of pheromone is given by parameters $\alpha$ and $\beta$, respectively. Generally, an off-line process must fit these parameters. Note that this probability distribution is affected by the parameters $\alpha$ and $\beta$, which determine the influence of the trails and the visibility, respectively. Finally, $S$ is the set of neighborhoods of node $i$, which have not visited yet by the ant $k$.

\subsection{Parameters $\alpha$ and $\beta$}

In particular, if $\alpha=0$, the nearest neighborhoods from a given node $i$ have more probability to be selected (well-known algorithm of the gradient with multiple starting points). If $\beta=0$, only the level of pheromone is considered for selecting the neighborhoods. This selection allows solutions with low quality respect to the three considered objective functions, specifically if $\alpha>1$. Indeed, this situation allows finding local optima of an ant algorithm, because all the ants follow the same way generating sub optimal solutions.

A proper updated pheromone matrix directly impacts the diversity on the decision-making process by an ant for selecting the following edges. A certain level of pheromone is deposited on an edge, when an ant has traveled across it. The level of pheromone is evaporated with the time. An additional positive feedback of the amount of pheromone is used to reinforce future components of good solutions. For a 
better solution, more pheromone is provided for the considered edges belonging to the best solutions found so far.

Eq. (5) determines the rule for increasing the quantity of pheromone

$$
\Delta \tau_{i j}^{k}=\left\{\begin{array}{cc}
\frac{1}{C\left(s_{k}\right)} & \text { if the edge }(i . j) \text { is used by the ant } k \text { on a performed route } \\
0 & \text { otherwise }
\end{array}\right.
$$

where $\Delta \tau_{i j}^{k}$ is the amount of pheromone deposited at the edges visited by the ant $k, C\left(s_{k}\right)$ is the total cost of the solution generated by the ant $k$, i.e. the total distance, time and energy consumed of the performed route by the ant $k$. The edges visited by all the ants in the current solution receive an extra contribution of pheromone.

In addition, a pheromone evaporation process is used to prevent an unlimited increasing of trails and to forget low quality solutions respect to the three objectives by the performed $k$ routes. The evaporation level is the same for all pheromone trails, eliminating a predefined percentage of the current value $\tau_{i j}(t)$ for each edge $(i . j)$, through a predefined rate $\rho$, with $0 \leq \rho \leq 1$. Eq. (6) describes the general rule for updating the pheromone matrix for all the edges $(i . j) \in A$, at each iteration $t$.

$$
\tau_{i j}(t+1)=(1-\rho) \tau_{i j}(t)+\sum_{A} \Delta \tau_{i j}^{k}
$$

\subsection{Ant System (AS)}

In the AS, an artificial colony of ants cooperates to find good solutions for discrete, static, and dynamic combinatorial optimization problems. Three different variants for the AS have been proposed: AntDensity, Ant-Quantity and Ant-Cycle (Wade \& Salhi, 2001). In the first two variants, the pheromoneupdated process is performed after each selection edge; while in the third variant (Ant-Cycle), the amount of pheromone is only updated once the ants have completed all the routes. The first two versions generally obtain worse results than the third version (Wade \& Salhi, 2001). Therefore, we consider the process for updating the pheromone by assigning a fixed amount of it on each edge at each iteration. Eq. (7) is generally used to determine the quantity of pheromone for each edge $(i, j)$, where $K$ is the number of ants, i.e. the number of available vehicles, and $C_{n n}$ is the tour length obtained at the constructive solution procedure.

$$
\tau_{i j}=\frac{K}{C_{n n}}
$$

\section{Proposed approach for the multi-objective MDVRPB}

The proposed approach is based on a Pareto Ant Colony Optimization (PACO). In particular, we have extended and enriched the main idea proposed by Doerner et al. (2004) for the problem of optimization of a financial portfolio to the Multi-objective Multi Depot Vehicle Routing Problem with Backhauls. The proposed approach take into consideration the rule described in Eq. (4). In this case, we have considered three matrices of pheromones for each objective function. These matrices are combined by using three objective weights $w_{d}$ (for the distance objective), $w_{t}$ (for the traveling time objective) and $w_{e}$ (for the objective function energy); i.e., the individual preferences of each ant, which are randomly initialized for each ant. The sum of the value of these factors must be equal to 1 . Note, that the domain of the three objective weights are random numbers equally distributed from the domain $[0,1)$. 
In the constructive initial solution, each ant tries to make a feasible route by applying a pseudo-random proportional rule using the heuristic information $\eta_{i j}$ and the pheromone information $\tau_{i j}(t)$. Once a feasible solution has been found, the three objective functions are calculated. If the solution $S$ is feasible, it is stored. The global pheromone updated process is performed by using the best solution found of the current iteration for each objective. The types of feasible solutions constructed are explained in the following section.

\subsection{Types of constructed routes}

Let us define the total number of depots as $m$ : i.e., $M_{1}, M_{2} \ldots M_{m}$, the total number of linehaul customers as $n l: L_{1}, L_{2}, \ldots, L_{n l}$ and the number of backhaul customers as $n b: B_{1}, B_{2}, \ldots, B_{n b}$. The proposed algorithm performs the following routes for a system with three (3) depots: $M_{1} . M_{2}$ and $M_{3}$; with seven (7) linehaul customers: $L_{1}, L_{2}, L_{3}, L_{4}, L_{5}, L_{6}$ and $L_{7}$; and seven (7) backhaul customers: $B_{1}, B_{2}, B_{3}, B_{4}, B_{5}, B_{6}$ and $B_{7}$ :

- A linehaul - backhaul route: The Fig. 1 describes this type of route. Its main feature is that the linehaul and the backhaul sequences are reaching the capacity of the vehicle.

- A single linehaul route: This route contains only linehaul customers. This route is shown in the Fig. 2.

- A single backhaul route: This type of route is depicted in the Fig. 3. It contains only backhaul customers.

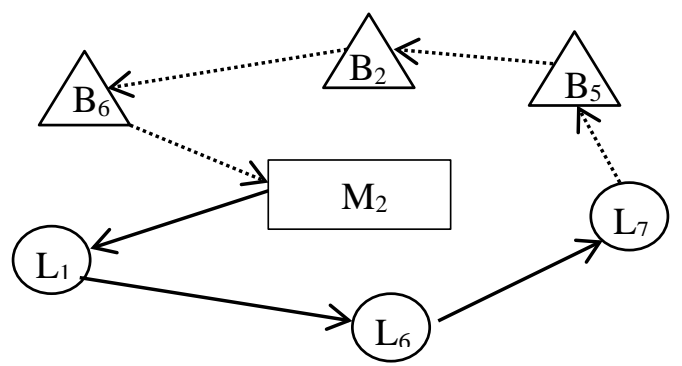

Fig. 1. Linehaul - backhaul route

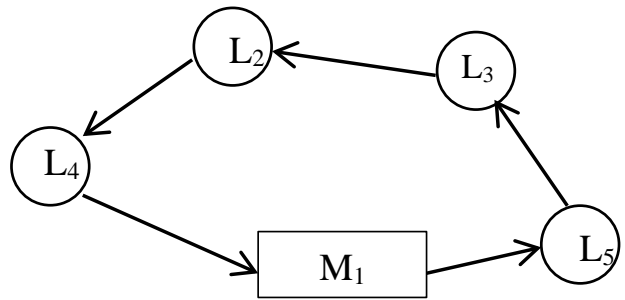

Fig. 2. Single linehaul route

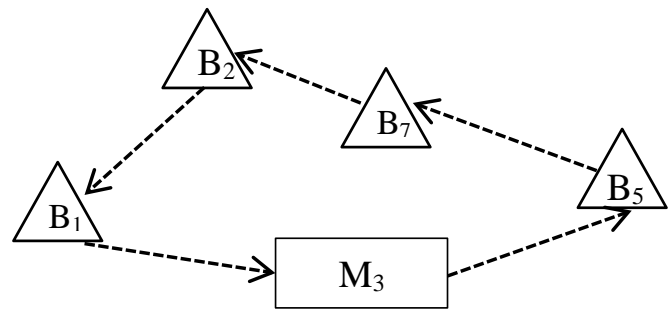

Fig. 3. Single backhaul route

An alternative of solution is conformed by a combination of the three described types of routes. In the proposed algorithm, the construction of a multi-depot solution requires that an ant performs as many subroutes as needed to visit all the customers, and each sub-route comprises a tour considering linehaul and backhaul customers. A possible alternative of solution is shown in the Fig. 4. The dashed line represents the entire tour performed by an ant. 


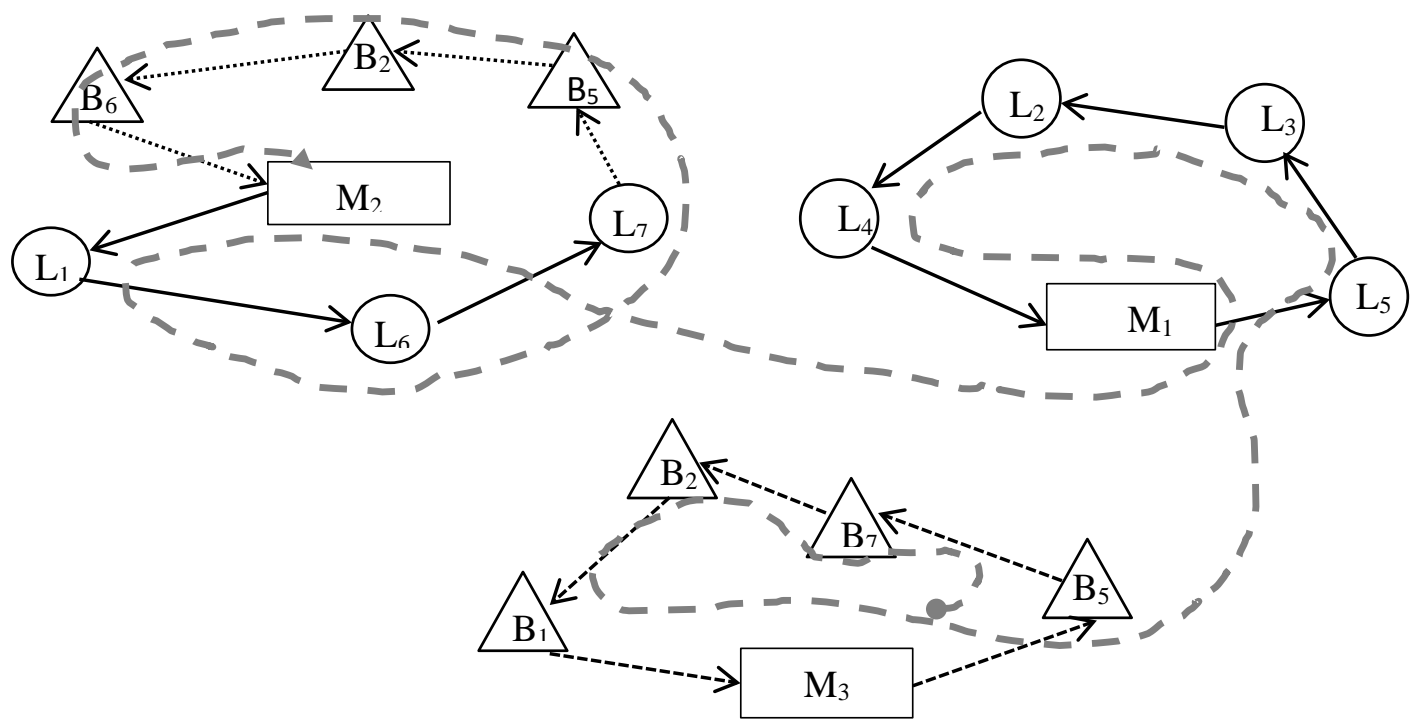

Fig. 4. Alternative route

\subsection{Decision rule}

Given the pheromone information, the set of objectives $o=\{1,2, \ldots, O\}$ and the set of all the feasible edges to be selected $\Omega(x, y)=\{(i, j) \in A\}$; the edge to be added to the current route performed by an ant is selected according to the following pseudo-random-proportional rule:

$$
(i, j)=\left\{\max _{(i, j) \in \Omega(x)}\left\{\left[\sum_{o=1}^{o} w_{o} \tau_{i j}\right]^{\alpha} \cdot \eta_{i j}{ }^{\beta}\right\} \quad \text { if } q<q_{0}\right.
$$

where $q$ is a random number between $[0,1), q_{0}$ is a given parameter between $[0,1)$ representing the probability to select the best next edge, and $\overline{(l, j)}$ is the edge to be selected according to the Eq. (4).

\subsection{Encoding Solution}

Fig. 5 shows the structure used for the distance, time and energy matrices. In particular, we have used a three-dimensional array with dimensions $(n+1) \times(n+1) \times(m)$ for each depot. For each matrix, each element $C_{i j}$ corresponds to the distance, the traveling time or the energy of the edge $(i . j)$.

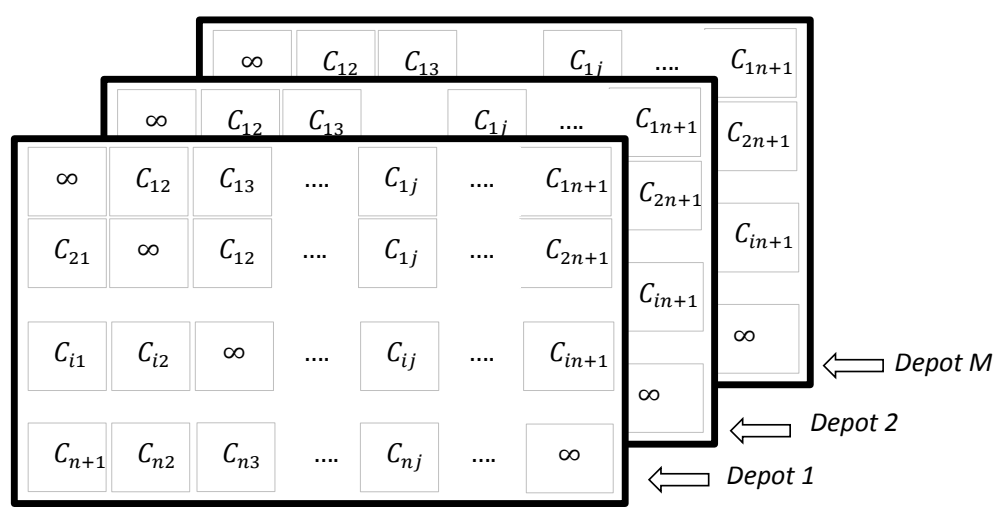

Fig. 5. Encoding for the distance, traveling time and energy matrices 
The Visibility Matrix (Fig. 6) is obtained from each element of the matrix elements of distance, time and energy. It contains the inverse matrix of the distance, time and energy. This matrix is symmetrical and is not modified during the execution of the algorithm.

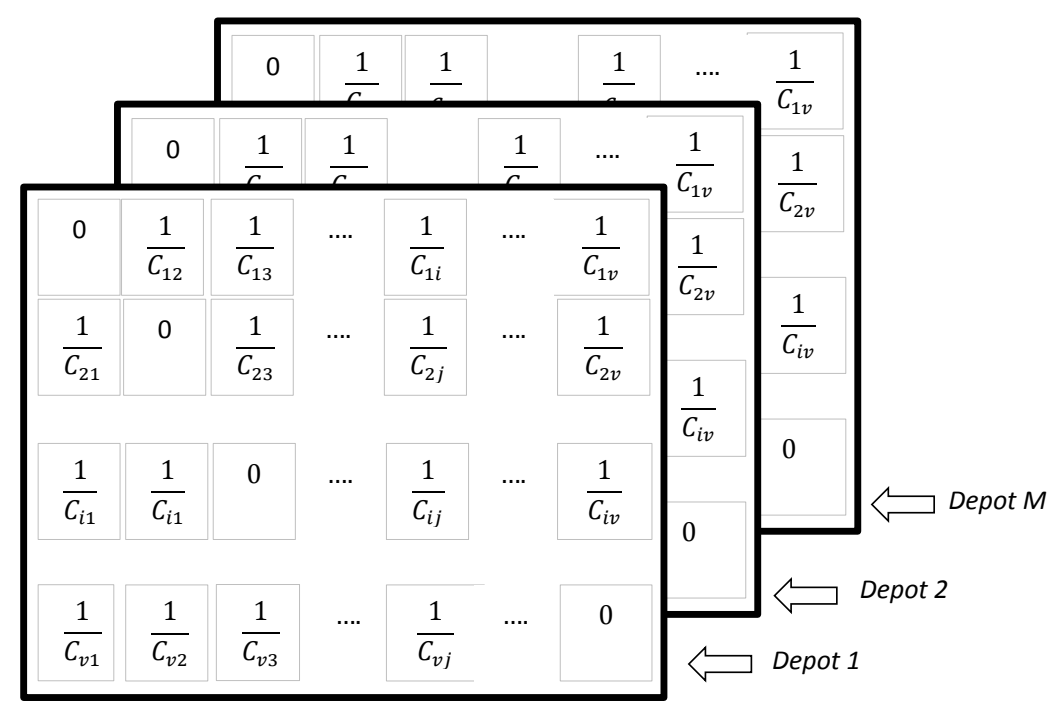

Fig. 6. Encoding of the Visibility matrix

Finally, we proceed to generate the pheromone matrix (Fig. 7) with all positions initialized with the same amount of pheromone. The values of the pheromone matrix are generated with the Eq. (7), and the updating process is restricted to thus elements in the route traveled by each ant.

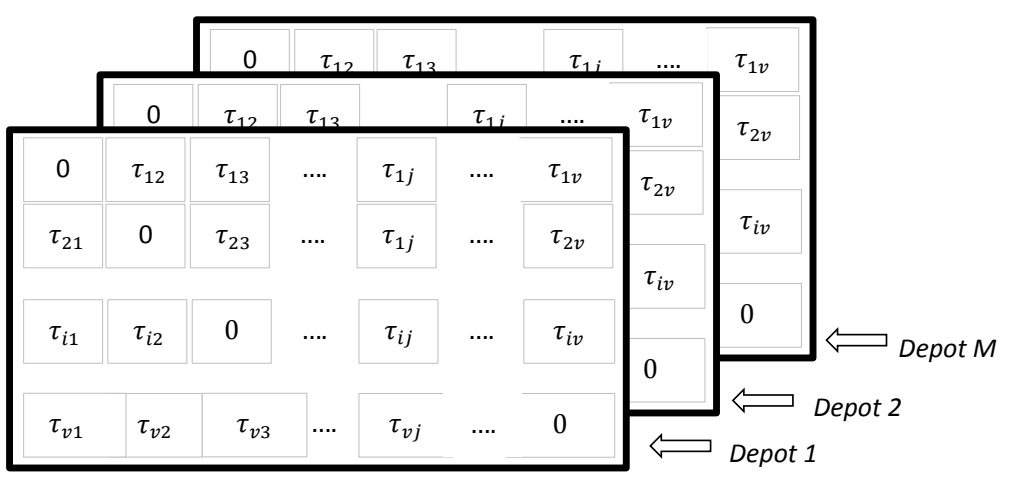

Fig. 7. Encoding the pheromone matrix

The proposed algorithm avoids flow between depots. Therefore, distance, time and energy among depots are not considered. Indeed, the proposed approach uses a new vehicle to continue visiting other customers. The visibility matrix or net matrix is replaced by a squared array of random values that provides diversity in the searching of the new depot to be added to the current solution. The total number of depots gives the dimension of the visibility matrix, and its values are initialized using the Eq. (7). The nodes are updated only if they belonging to the route traveled by a vehicle. In addition, a vector of not visited customers is required. It contains the feasible customers to be selected.

The solution vector is also required. Its first element is the number of a depot, which is randomly selected. It is followed by a sequence of customer until a new depot has been selected again. However, for each sequence of customers, a Linehaul customer must not appear between two Backhaul customers, and vice versa. The Figure 8 shows an example of a route of the proposed solution. 


\begin{tabular}{|c|c|c|c|c|c|c|c|c|c|c|c|c|c|c|c|c|c|}
\hline $\mathrm{M}_{2}$ & $\mathrm{~L}_{1}$ & $\mathrm{~L}_{6}$ & $\mathrm{~L}_{7}$ & $\mathrm{~B}_{5}$ & $\mathrm{~B}_{1}$ & $\mathrm{~B}_{6}$ & $\mathrm{M}_{2}$ & $\mathrm{M}_{1}$ & $\mathrm{~L}_{4}$ & $\mathrm{~L}_{2}$ & $\mathrm{M}_{1}$ & $\mathrm{M}_{3}$ & $\mathrm{~B}_{4}$ & $\mathrm{~B}_{3}$ & $\mathrm{~B}_{2}$ & $\mathrm{~B}_{7}$ & $\mathrm{M}_{3}$ \\
\hline
\end{tabular}

Fig. 8. Encoding the solution Vector

\subsection{Pseudocode for the proposed algorithm}

Procedure PACO-MDVRPB (depots. customers. vehicleNumber. vehicleCapacity)

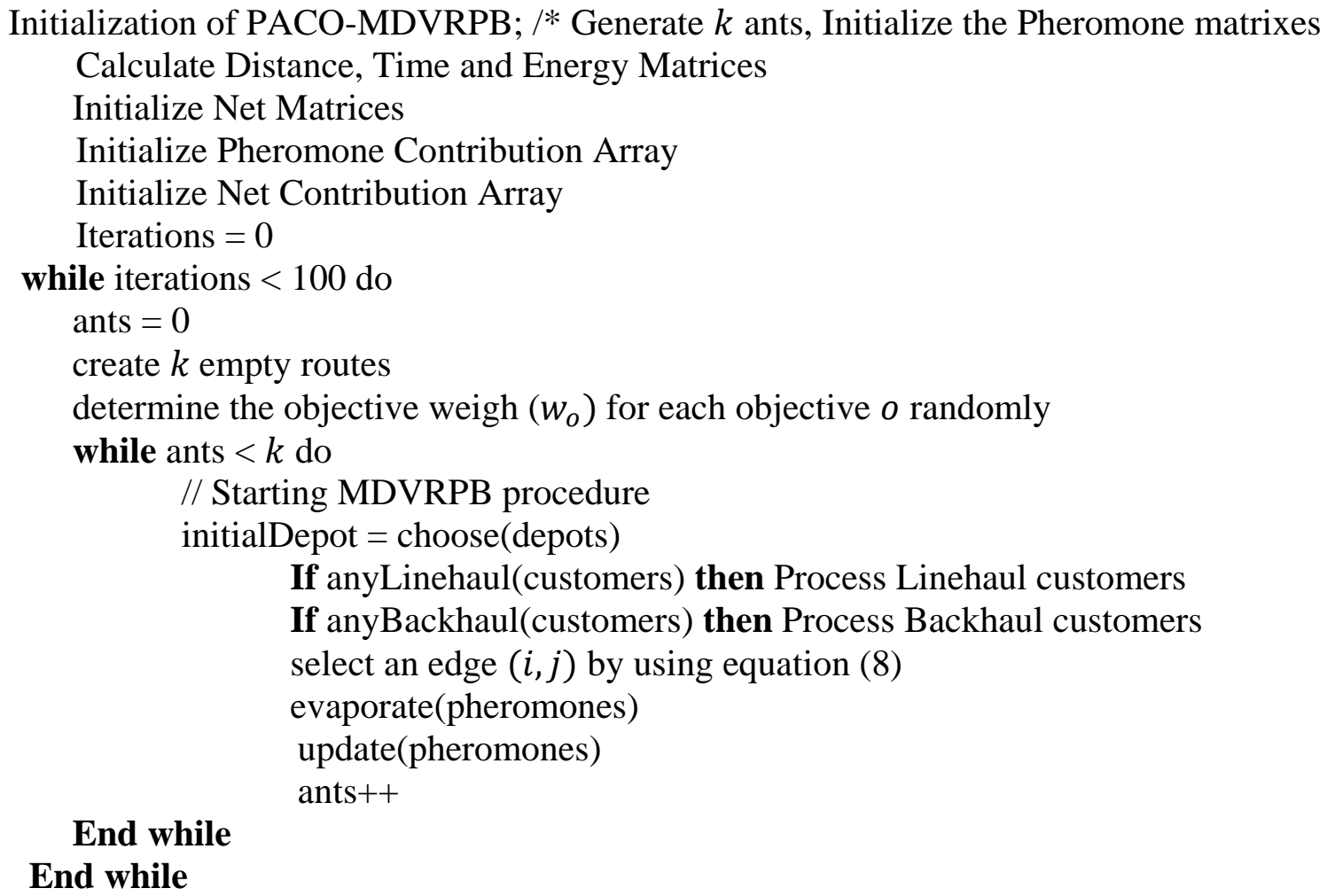

\section{Computational results}

The proposed approach has been tested on an adapted benchmarking set, which is available in http://unilibrepereira.edu.co/backhauls/. In particular, 33 benchmarking instances are used to test the effectiveness of the proposed approach. The proposed algorithm has been implemented in MatLab, and computational experiments have been run on a PC with Core i5 $1.4 \mathrm{GHz}$ processor and 8GB of RAM. The algorithm has been executed for 10 runs over 100 iterations, reporting the average results and the best results of all runs.

The parameters used to execute the complete set of instances are the following: Iterations $=100, \alpha=1, \beta$ $=3, \rho=0.01$, and the contribution factor of the visited edge $=$ Number of customers $/$ Best solution found so far.

\subsection{Description of the Instances}

The proposed algorithm has been evaluated in 33 instances MDVRPB adapted from Salhi and Nagy (1999). The respective set has a diverse number of Linehaul and Backhaul customers (from 50 to 250), a number of depots between $2-5$, and a homogeneous set of vehicles are considered. Euclidean distance for each edge is determined. The set of benchmarking instances has been used to solve different variants of the MDVRPB. However in the reviewed literature, we have not found papers that solve the problem MDVRPB with Backhauls at the end of the routes. 
The matrices of energy have been performed as follows: We have defined three types of vehicles depending of the load (Type 1-T1 with less than 10 ton, Type 2-T2 between 10 and 20 ton, and Type 3T3 between 20 and 35 ton). The parameters of the frontal area of each vehicle, weigh of the vehicle, weigh of the load, values of $C_{d}$ and $C_{r}$ are described in Table 1.

Table 1

Energy parameters (Source: http://www.kenworthcolombia.com/)

\begin{tabular}{cccc}
\hline & Type 1-T1 & Type 2-T2 & Type 3-T3 \\
\hline Frontal Area $\left.\mathbf{( m}^{\mathbf{2}}\right)$ & 7 & 9 & 11.44 \\
Weight of the vehicle $(\mathbf{k g})$ & 6000 & 16000 & 17000 \\
Weight of the load $(\mathbf{k g})$ & 10000 & 20000 & 35000 \\
$\boldsymbol{C}_{\boldsymbol{d}}$ & 0.76 & 0.85 & 0.95 \\
$\boldsymbol{C}_{\boldsymbol{r}}$ & 0.01 & 0.0125 & 0.015 \\
\hline
\end{tabular}

In addition, the following parameters are considered to obtain the energy matrices: acceleration $=0 \mathrm{~m} / \mathrm{s} 2$, gravity $=9.807$, angle of the road $=0^{\circ}$, air density at $20^{\circ} \rho=1.2041 \mathrm{~kg} / \mathrm{m}^{3}$. Finally, the distances and the speed for each edge are calculated with the coordinates $X$ and $Y$ and the matrices of traveling time. Table 2 shows the type of vehicle used for each instance.

Table 2

Type of used vehicle for each instance

\begin{tabular}{|c|c|c|c|}
\hline Instance Number & Instance & Vehicle Capacity (units) & Type of Vehicle \\
\hline $1,2,3$ & GJ01H, GJ01Q, GJ01T & 80 & 1 \\
\hline $4,5,6,7,8,9$ & $\begin{array}{l}\text { GJ02H, GJ02Q, GJ02T } \\
\text { GJ03H, GJ03Q,GJ03T }\end{array}$ & 160 & 2 \\
\hline $\begin{array}{c}10,11,12,16,17,18,19,20 \text {, } \\
21\end{array}$ & $\begin{array}{l}\text { GJ04H, GJ04Q, GJ04T, } \\
\text { GJ06H, GJ06Q, GJ05T, } \\
\text { GJ06Q, GJ06T, GJ07H, } \\
\text { GJ07Q, GJ07T }\end{array}$ & 100 & 1 \\
\hline $13,14,15$ & GJ05H, GJ05Q, GJ05T & 200 & 2 \\
\hline $\begin{array}{c}22,23,24,25,26,27,28,29 \\
30,31,32,33\end{array}$ & $\begin{array}{l}\text { GJ08H, GJ08Q, GJ08T, } \\
\text { GJ09H, GJ09Q, GJ09T, } \\
\text { GJ10H, GJ10Q, GJ10T, } \\
\text { GJ11H, GJ11Q, GJ11T }\end{array}$ & 500 & 3 \\
\hline
\end{tabular}

In addition, the correlation of the objectives for each instance is calculated in order to justify the consideration of the three objectives at the same time. As an example, Fig. 9, Fig. 10 and Fig. 11 show the correlation of the three objectives for instance 3. Note that there are low correlations factors for the considered objectives. Therefore, the objectives are independent and a Pareto Front must be calculated.

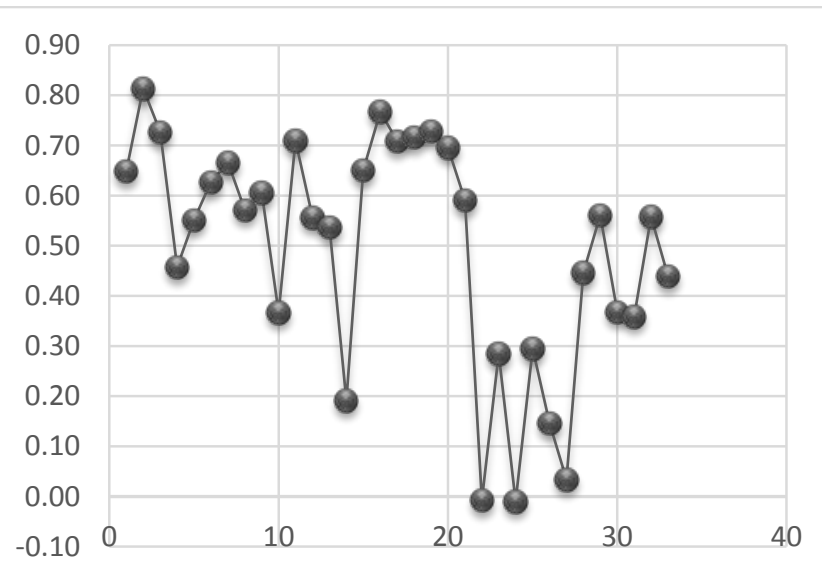

Fig. 9. Correlation between Distance and Time

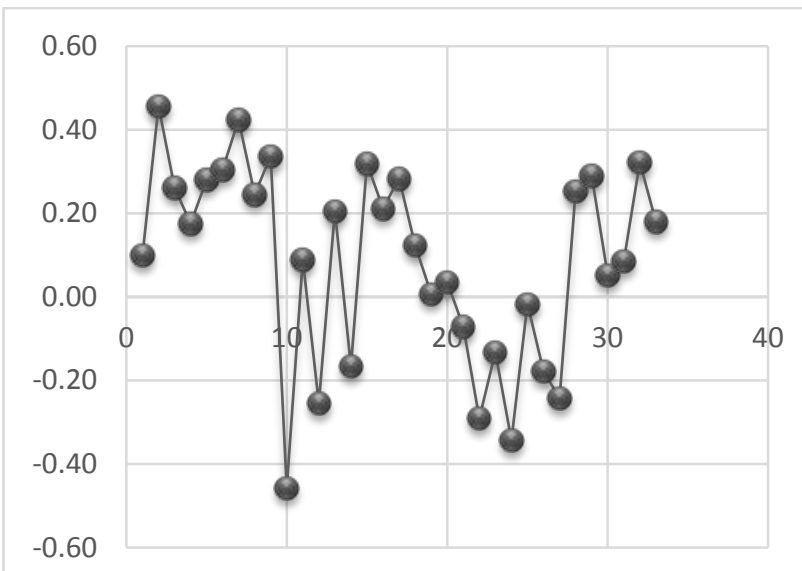

Fig.10. Correlation between Time and Energy 


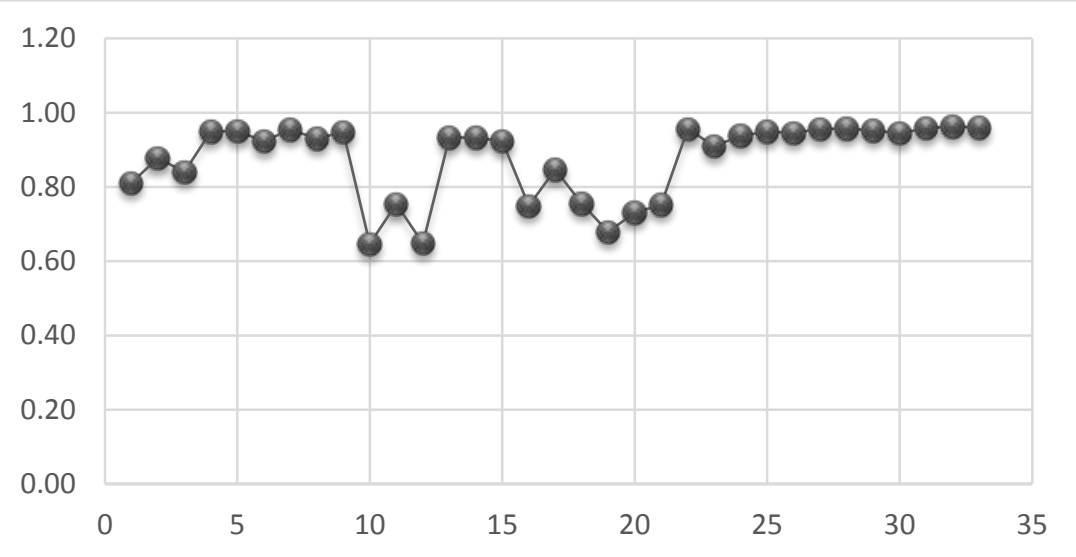

Fig. 11. Correlation between Distance and Energy

\subsection{Obtained Results}

We report the computing time and the values reached by the metaheuristic objective proposed

Table 3

Obtained results over 10 executions with 100 iterations of the proposed algorithm

\begin{tabular}{|c|c|c|c|c|c|c|c|c|c|c|c|c|c|}
\hline \multirow[b]{2}{*}{$\begin{array}{l}\text { Instance } \\
\text { Number }\end{array}$} & \multirow[b]{2}{*}{ Instance } & \multicolumn{4}{|c|}{$\begin{array}{c}\text { Proposed Methodology with PACO - } \\
\text { Distance }\end{array}$} & \multicolumn{4}{|c|}{$\begin{array}{c}\text { Proposed Methodology with PACO - } \\
\text { Time }\end{array}$} & \multicolumn{4}{|c|}{$\begin{array}{l}\text { Proposed Methodology with PACO - } \\
\text { Energy }\end{array}$} \\
\hline & & $\begin{array}{c}\text { Best } \\
\text { Solution } \\
\text { Distance } \\
(\mathbf{k m})\end{array}$ & $\begin{array}{c}\text { Objective } \\
\text { Function } \\
\text { Time } \\
\text { (min) }\end{array}$ & $\begin{array}{c}\text { Objective } \\
\text { Function } \\
\text { Energy } \\
\text { (kw/h) }\end{array}$ & $\begin{array}{c}\text { Computing } \\
\text { Time } \\
\text { (sec) }\end{array}$ & $\begin{array}{c}\text { Objective } \\
\text { Function } \\
\text { Distance } \\
(\mathbf{k m})\end{array}$ & $\begin{array}{c}\text { Best } \\
\text { Solution } \\
\text { Time } \\
\text { (min) }\end{array}$ & $\begin{array}{c}\text { Objective } \\
\text { Function } \\
\text { Energy } \\
\text { (kw/h) }\end{array}$ & $\begin{array}{c}\text { Computing } \\
\text { Time } \\
\text { (sec) }\end{array}$ & $\begin{array}{c}\text { Objective } \\
\text { Function } \\
\text { Distance } \\
(\mathbf{k m})\end{array}$ & $\begin{array}{c}\text { Objective } \\
\text { Function } \\
\text { Time } \\
\text { (min) }\end{array}$ & $\begin{array}{c}\text { Best } \\
\text { Solution } \\
\text { Energy } \\
\text { (kw/h) }\end{array}$ & $\begin{array}{c}\text { Computing } \\
\text { Time } \\
\text { (sec) }\end{array}$ \\
\hline 1 & GJ01H & 674.6 & 828.9 & 449.5 & 3.57 & 804.6 & 716 & 646.6 & 2.64 & 675.7 & 833.7 & 446.2 & 2.46 \\
\hline 2 & GJ01Q & 776.5 & 868.9 & 528.9 & 3.33 & 865.1 & 790.6 & 669.5 & 2.49 & 802.1 & 977.8 & 512.9 & 2.42 \\
\hline 3 & GJ01T & 762.6 & 848.9 & 533.2 & 2.9 & 886.4 & 751.7 & 728 & 1.38 & 771.5 & 922.9 & 522.1 & 1.56 \\
\hline 4 & GJ02H & 639.4 & 675.8 & 1034 & 1.06 & 739.6 & 651.7 & 1280.4 & 1.73 & 651.8 & 732.3 & 1022.7 & 0.75 \\
\hline 6 & GJ02T & 678.2 & 698.6 & 1124.4 & 2.4 & 744.2 & 632.8 & 1314.8 & 2.57 & 680.7 & 743.7 & 1077.2 & 1.57 \\
\hline 7 & GJ03H & 836.5 & 969 & 1322.5 & 4.82 & 858.8 & 820.3 & 1465.1 & 4.36 & 840.3 & 982.2 & 1321.2 & 2.05 \\
\hline 8 & GJ03Q & 929 & 994.7 & 1499.2 & 3.29 & 984.4 & 907.2 & 1692.4 & 6.16 & 948.9 & 1150.1 & 1463.1 & 3.7 \\
\hline 9 & GJ03T & 909.5 & 1060.1 & 1416.1 & 5.65 & 956.4 & 911.6 & 1645.4 & 6.61 & 916 & 1096.7 & 1409.9 & 2.87 \\
\hline 10 & GJ04H & 1203 & 1307.3 & 845.6 & 3.46 & 1360.5 & 1170.4 & 1109.7 & 2.7 & 1226.5 & 1536.7 & 778.6 & 3.94 \\
\hline 11 & GJ04Q & 1287.2 & 1492.1 & 870.2 & 2.93 & 1439.2 & 1357.4 & 1116.3 & 2.76 & 1312.8 & 1647.9 & 840.5 & 0.98 \\
\hline 12 & GJ04T & 1269.3 & 1515.5 & 848 & 1.41 & 1391.8 & 1258.2 & 1087.8 & 3.6 & 1276.2 & 1563.2 & 835.8 & 1.36 \\
\hline 13 & GJ05H & 992.4 & 1055.2 & 1639.9 & 1.62 & 1096.1 & 987.7 & 1909.7 & 2.14 & 1024.5 & 1195.7 & 1619.9 & 2.05 \\
\hline 14 & GJ05Q & 1063.5 & 1245.1 & 1664.2 & 3.19 & 1124.5 & 1083.9 & 1901 & 1.71 & 1076.3 & 1303.6 & 1656.7 & 1.8 \\
\hline 16 & GJ06H & 1148.1 & 1199.6 & 838.4 & 4.63 & 1268.2 & 1120.9 & 1011.9 & 3.12 & 1222 & 1564.2 & 770 & 3.79 \\
\hline 17 & GJ06Q & 1205.6 & 1223 & 878.7 & 6.73 & 1269.3 & 1117.2 & 1011.4 & 5.8 & 1301.2 & 1540 & 867.6 & 3.53 \\
\hline 18 & GJ06T & 1203 & 1423.4 & 803.1 & 6.65 & 1274 & 1207.6 & 963.6 & 3.71 & 1240.7 & 1576 & 784.7 & 3.28 \\
\hline 19 & GJ07H & 1100.6 & 1142.7 & 808.6 & 6.32 & 1231.9 & 1070.6 & 995.3 & 4.75 & 1144.4 & 1445 & 736.6 & 2.74 \\
\hline 20 & GJ07Q & 1198.6 & 1392.4 & 817.9 & 8.64 & 1332.7 & 1151.6 & 1083.7 & 4.33 & 1200.4 & 1397.7 & 807.2 & 5.69 \\
\hline 21 & GJ07T & 1196 & 1364.4 & 824.3 & 7.86 & 1315.1 & 1174.6 & 1049.4 & 4.91 & 1200.3 & 1524.9 & 758.9 & 6.68 \\
\hline 22 & GJ08H & 5114.7 & 5933.9 & 13481.2 & 11 & 5432 & 4858.5 & 15515.4 & 13.88 & 5154.8 & 6091.3 & 13457.3 & 6.9 \\
\hline 23 & GJ08Q & 5763.4 & 5835.7 & 15757.3 & 20.36 & 6119.5 & 5473.9 & 17584.9 & 18.8 & 5904.2 & 6819.7 & 15527.1 & 14.38 \\
\hline 24 & GJ08T & 5879 & 5779.8 & 16475.1 & 24.54 & 5984.5 & 5593 & 17030.9 & 13.42 & 5936.9 & 6953.6 & 15472.4 & 13.42 \\
\hline 25 & GJ09H & 5106.8 & 5624.9 & 13805.7 & 13.9 & 5160.7 & 4707.6 & 14694.2 & 12.53 & 5112.1 & 5665.7 & 13802.7 & 9.3 \\
\hline 26 & GJ09Q & 5889.9 & 6279.6 & 16017.6 & 11.45 & 6571.8 & 5759.2 & 19018.3 & 12.2 & 5906.8 & 6365 & 15968 & 10.99 \\
\hline 27 & GJ09T & 6135.2 & 6955.2 & 16281.4 & 15.31 & 6505.4 & 6152.4 & 18347.4 & 15.68 & 6325.3 & 7989.1 & 16064 & 10.28 \\
\hline 28 & GJ10H & 4814.2 & 5458.5 & 12859.8 & 18.14 & 4888.1 & 4749 & 13759.5 & 21.65 & 4959.8 & 5865.1 & 12808.1 & 17.03 \\
\hline 29 & GJ10Q & 5387.3 & 5727.3 & 14550.6 & 24.91 & 6274.3 & 5434.1 & 18245.8 & 30.17 & 5596.6 & 6732.9 & 14453.3 & 28.9 \\
\hline 30 & GJ10T & 5305.4 & 5914 & 14201.1 & 55.1 & 5458.3 & 5249.7 & 15321.9 & 27.53 & 5379.6 & 6535 & 13896.9 & 33.05 \\
\hline 31 & GJ11H & 4725.9 & 5226.8 & 12664 & 28.01 & 5286.7 & 4768.7 & 15107.8 & 25.88 & 4725.9 & 5226.8 & 12664 & 24.59 \\
\hline 32 & GJ11Q & 5279.7 & 5716.9 & 14208.3 & 57.18 & 5882.5 & 5293.2 & 16788.1 & 50.16 & 5366.7 & 6500.5 & 13804.1 & 22.91 \\
\hline 33 & GJ11T & 5149.7 & 5577.4 & 13862.7 & 47.72 & 5629.9 & 5040 & 16203.9 & 50.63 & 5184.4 & 5836.2 & 13715.6 & 18.85 \\
\hline
\end{tabular}

Fig. 12, Fig. 13 and Fig. 14 show the results found for the instance 3 of the Pareto Fronts by using the criterion Min - Min. Note that each figure shows the extreme values of each Pareto Front. 


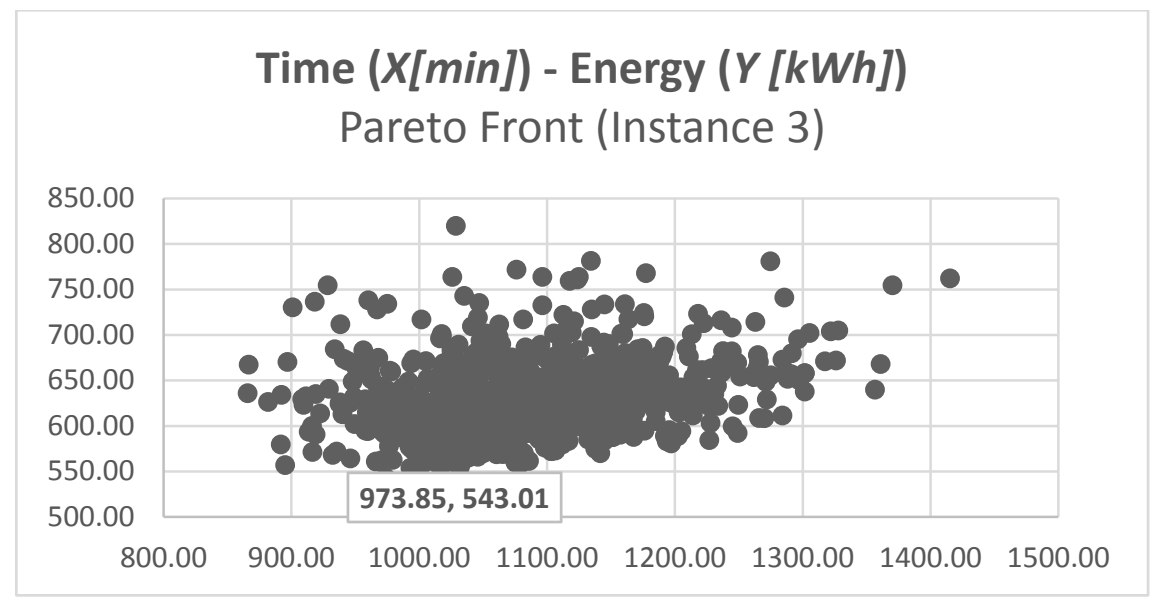

Fig. 12. Pareto Ant Colony Optimization: Time - Energy

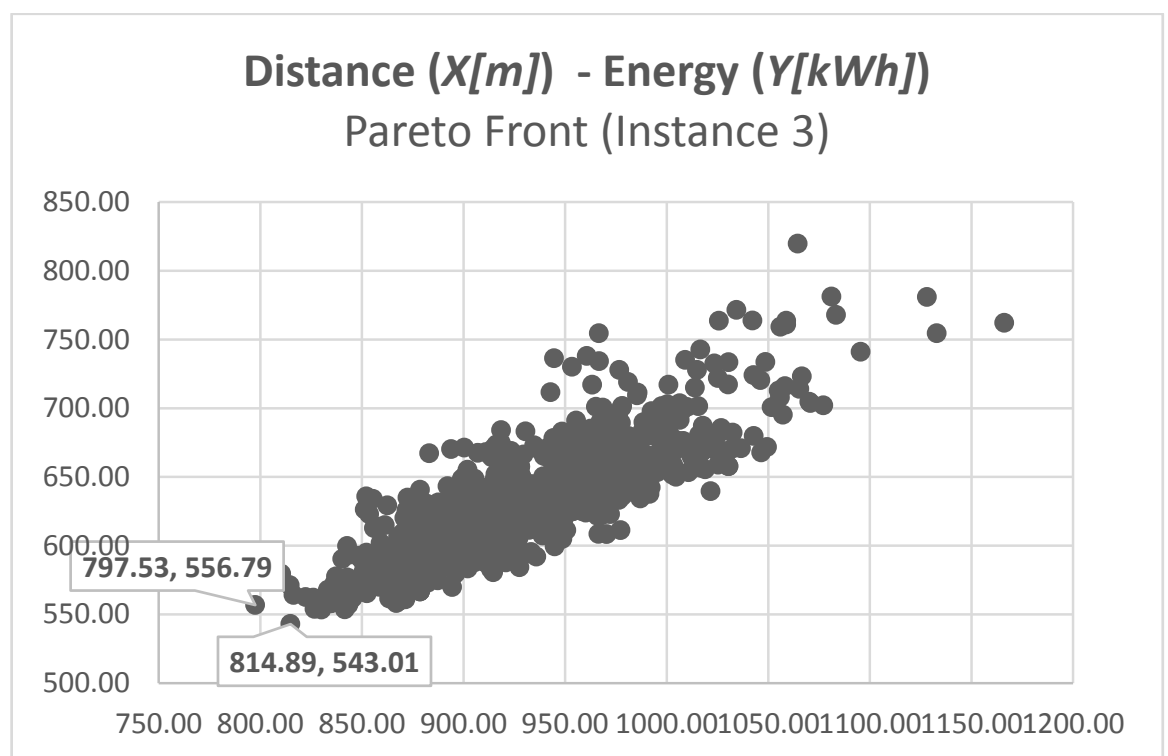

Fig. 13. Pareto Ant Colony Optimization: Distance - Energy

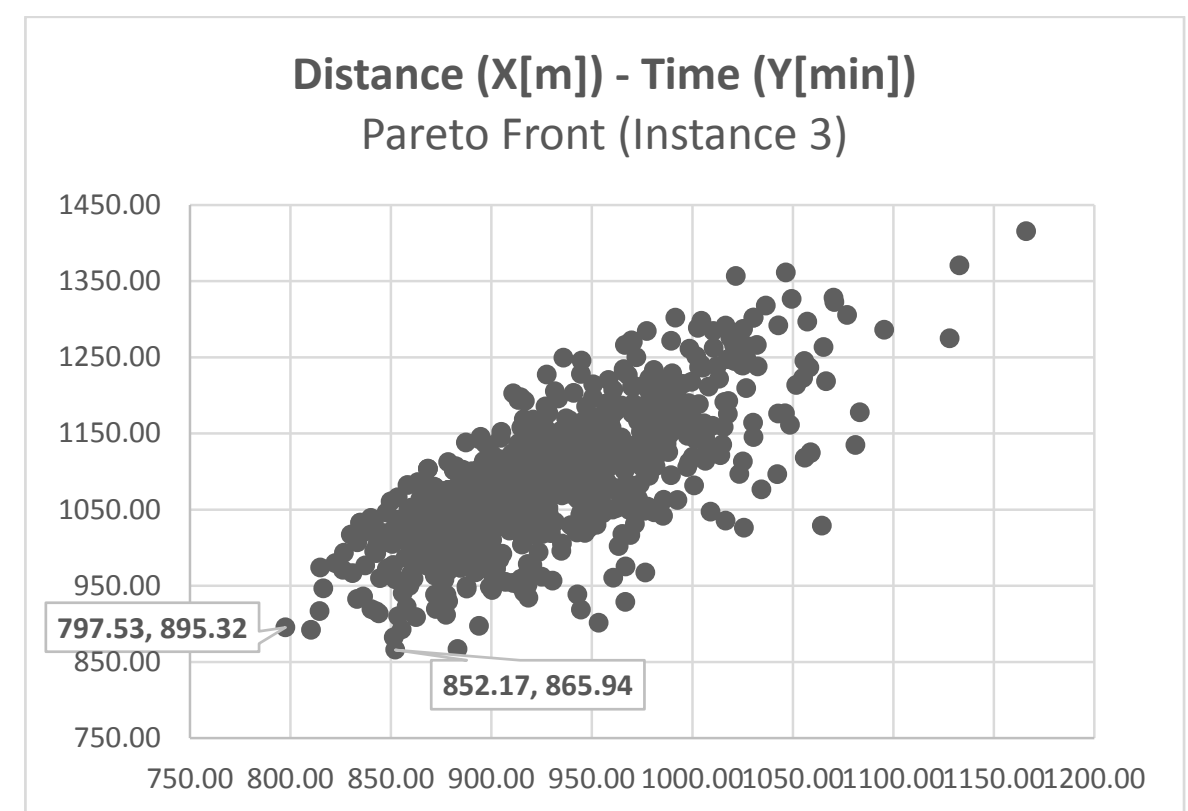

Fig. 14. Pareto Ant Colony Optimization: Distance - Time 


\section{Concluding remarks}

Multi-objective combinatorial techniques play a decisive role in the field of the vehicle routing problems. Recent researchers have proposed several approaches to solve several variants of the multi-objective vehicle routing problems classified as NP-hard problems.

In this paper, an effective Pareto Ant Colony Optimization has been used to provide an efficient approach for the Multi-objective Multi-Depot Vehicle Routing Problem with Backhauls (MDVRPB). In particular, three objectives of traveled distance, traveling times and total consumption of energy have been minimized. In addition, we have used multiple pheromone matrices and random weights for each objective. The performance of the proposed algorithm has been evaluated by considering adapted instances from the literature.

The proposed methodology could be extended to other vehicle routing problems with many or few constraints and/or objectives. In addition, new heuristic information could be added easily to the proposed approach. We suggest proving the proposed approach to other routing problems such as the Multi Depot Vehicle Routing Problem (MDVRP), the Periodic Location Routing Problem (PLRP), the Muti-Depot Vehicle Routing Problem with Heterogeneous Fleet (HMDVRP), among others.

\section{References}

Anbuudayasankar, S. P., Ganesh, K., Koh, S. L., \& Ducq, Y. (2012). Modified savings heuristics and genetic algorithm for bi-objective vehicle routing problem with forced backhauls. Expert Systems with Applications, 39(3), 2296-2305.

Bektaş, T., \& Laporte, G. (2011). The pollution-routing problem. Transportation Research Part B: Methodological, 45(8), 1232-1250.

Bolaños, R., Echeverry, M., \& Escobar, J. (2015). A multiobjective non-dominated sorting genetic algorithm (NSGA-II) for the Multiple Traveling Salesman Problem. Decision Science Letters, 4(4), 559-568.

Chunyu, R., Zhendong, S., \& Xiaobo, W. (2009, June). Study on single and mixed fleet strategy for multi-depot vehicle routing problem with backhauls. In Computational Intelligence and Natural Computing, 2009. CINC'09. International Conference on (Vol. 1, pp. 425-428). IEEE.

Chunyu, R., \& Xiaobo, W. (2009, October). Study on hybrid genetic algorithm for multi-type vehicles and multi-depot vehicle routing problem with backhauls. In Intelligent Computation Technology and Automation, 2009. ICICTA'09. Second International Conference on (Vol. 1, pp. 197-200). IEEE.

Demir, E., Bektaş, T., \& Laporte, G. (2014). The bi-objective pollution-routing problem. European Journal of Operational Research, 232(3), 464-478.

Doerner, K., Gutjahr, W. J., Hartl, R. F., Strauss, C., \& Stummer, C. (2004). Pareto ant colony optimization: A metaheuristic approach to multiobjective portfolio selection. Annals of Operations Research, 131(1-4), 79-99.

Escobar, J. W., Linfati, R., \& Toth, P. (2013). A two-phase hybrid heuristic algorithm for the capacitated location-routing problem. Computers \& Operations Research, 40(1), 70-79.

Escobar, J. W., Linfati, R., Toth, P., \& Baldoquin, M. G. (2014a). A hybrid granular tabu search algorithm for the multi-depot vehicle routing problem. Journal of Heuristics, 20(5), 483-509.

Escobar, J. W., Linfati, R., Baldoquin, M. G., \& Toth, P. (2014b). A Granular Variable Tabu Neighborhood Search for the capacitated location-routing problem. Transportation Research Part B: Methodological, 67, 344-356.

Escobar, J. W., Linfati, R., \& Adarme-Jaimes, W. (2015). A hybrid metaheuristic algorithm for the capacitated location routing problem. Dyna, 82(189), 243-251.

García-Nájera, A., Bullinaria, J. A., \& Gutiérrez-Andrade, M. A. (2015). An evolutionary approach for multi-objective vehicle routing problems with backhauls. Computers \& Industrial Engineering, 81, 90-108. 
Gutjahr, W. J. (2002). ACO algorithms with guaranteed convergence to the optimal solution. Information Processing Letters, 82(3), 145-153.

Jozefowiez, N., Semet, F., \& Talbi, E. G. (2008). Multi-objective vehicle routing problems. European Journal of Operational Research, 189(2), 293-309.

Lau, H. C., Chan, T. M., Tsui, W. T., Chan, F. T., Ho, G. T., \& Choy, K. L. (2009). A fuzzy guided multi-objective evolutionary algorithm model for solving transportation problem. Expert Systems with Applications, 36(4), 8255-8268.

Liu, C. M., Chang, T. C., \& Huang, L. F. (2006). Multi-objective heuristics for the vehicle routing problem. International Journal of Operations Research, 3(3), 173-181.

Min, H., Current, J., \& Schilling, D. (1992). The multiple depot vehicle routing problem with backhauling. Journal of Business Logistics, 13(1), 259.

Mohammadi, M., Tavakkoli-Moghaddam, R., \& Rostami, R. (2011). A multi-objective imperialist competitive algorithm for a capacitated hub covering location problem. International Journal of Industrial Engineering Computations, 2(3), 671-688.

Mortezaei, M., \& JabalAmeli, M. (2011). A hybrid model for multi-objective capacitated facility location network design problem. International Journal of Industrial Engineering Computations, 2(3), 509524.

Nezhad, A., Roghanian, E., \& Azadi, Z. (2013). A fuzzy goal programming approach to solve multiobjective supply chain network design problems. International Journal of Industrial Engineering Computations, 4(3), 315-324.

Rao, R., \& Patel, V. (2014). A multi-objective improved teaching-learning based optimization algorithm for unconstrained and constrained optimization problems. International Journal of Industrial Engineering Computations, 5(1), 1-22.

Ropke, S., \& Pisinger, D. (2006). A unified heuristic for a large class of vehicle routing problems with backhauls. European Journal of Operational Research, 171(3), 750-775.

Salhi, S., \& Nagy, G. (1999). A cluster insertion heuristic for single and multiple depot vehicle routing problems with backhauling. Journal of the Operational Research Society, 50(10), 1034-1042.

Wade, A., \& Salhi, S. (2001, July). An ant system algorithm for the vehicle routing problem with backhauls. In MICÕ2001-4th Metaheuristic International Conference.

Wade, A., \& Salhi, S. (2004). An ant system algorithm for the mixed vehicle routing problem with backhauls. In Metaheuristics: computer decision-making (pp. 699-719). Springer US.

Yalcın, G. D., \& Erginel, N. (2015). Fuzzy multi-objective programming algorithm for vehicle routing problems with backhauls. Expert Systems with Applications, 42(13), 5632-5644.

Yazdian, S., \& Shahanaghi, K. (2011). A multi-objective possibilistic programming approach for locating distribution centers and allocating customers demands in supply chains. International Journal of Industrial Engineering Computations, 2(1), 193-202.

Zhou, A., Qu, B. Y., Li, H., Zhao, S. Z., Suganthan, P. N., \& Zhang, Q. (2011). Multiobjective evolutionary algorithms: A survey of the state of the art. Swarm and Evolutionary Computation, 1(1), 32-49. 